\title{
Controlled engineering of spin polarized transport properties in a zigzag graphene nanojunction
}

\author{
Sudin Ganguly ${ }^{1}$, Saurabh Basu ${ }^{1}$ and Santanu K. Maiti ${ }^{2}$ \\ Department of Physics, Indian Institute of Technology Guwahati, Guwahati-781039, Assam, India \\ Physics and Applied Mathematics Unit, Indian Statistical Institute, 203 Barrackpore Trunk Road, Kolkata-700 108, \\ India \\ PACS 72.80.Vp - Electronic transport in graphene \\ PACS 72.25.-b - Spin polarized transport \\ PACS 73.63.-b - Electronic transport in nanoscale materials and structures

\begin{abstract}
We investigate a novel way to manipulate the spin polarized transmission in a two terminal zigzag graphene nanoribbon in presence of Rashba spin-orbit (SO) interaction with circular shaped cavity engraved into it. A usual technique to control the spin polarized transport behaviour of a nanoribbon can be achieved by tuning the strength of the SO coupling, while we show that an efficient engineering of the spin polarized transport properties can also be done via cavities of different radii engraved in the nanoribbon. Simplicity of the technique in creating such cavities in the experiments renders an additional handle to explore transport properties as a function of the location of the cavity in the nanoribbon. Further, a systematic assessment of the interplay of the Rashba interaction and the dimensions of the nanoribbon is presented. These results should provide useful input to the spintronic behaviour of such devices. In addition to the spin polarization, we have also included an interesting discussion on the charge transmission properties of the nanoribbon, where, in absence of any SO interaction a metal-insulator transition
\end{abstract} \\ induced by the presence of a cavity is observed.
}

Introduction. - Spin-based electronics or spintronics is one of the most promising field for future powerconsuming high operating speed, new forms of information storage and logic devices [1]. The key factor for the development of spin-based electronics [2] is the fine control of the spin-polarized current. For this purpose, a major challenge is developing a suitable spin transport channel with long spin diffusion length and spin lifetime. Graphene 3, believed to be a very promising candidate in the spintronic applications, owing to the achievement of room-temperature spin transport with long spin-diffusion lengths (up to $\sim 100 \mu \mathrm{m}$ ) 4] 8. Graphene has also several interesting electronic and transport properties [9], that make it very attractive for spintronic applications, such as quasirelativistic band structure [3, 10, unconventional quantum Hall effect [3, 10, 11, half metallicity [12,13] and high carrier mobility [14,15].

Recent experimental realization of freestanding graphene nanoribbons (GNRs) [16, 17] has generated renewed interest in carbon-based materials with exotic properties. GNRs are basically single strips of graphene where electronic properties [18, 19 depend on the geometry of the edges and the lateral width of the nanoribbons 20. Depending upon the edge structures, GNRs can be of two types, namely armchair graphene nanoribbon (AGNR) and zigzag graphene nanoribbon (ZGNR). Irrespective of the width of the ZGNR, they are always metallic with zero bandgap, while the AGNRs are conditionally metallic, that is when the lateral width $N_{y}=3 M-1$ ( $M$ is an integer), else the AGNRs are semiconducting in nature [18 with a finite band gap. GNR is also known to have long spin-diffusion length, spin relaxation time, and electron spin coherence time 21 23.

SO coupling (SOC) plays a crucial role in spintronic devices. Two kinds of SOC can be present in graphene, the intrinsic SOC and the Rashba SOC (RSOC) [24, 25. The strength of the intrinsic SOC is negligibly small in pristine graphene (up to $\sim 0.01-0.05 \mathrm{meV}$ ) [26, 27]. On the other hand, the strength of the Rashba SOC can be modulated by an external electric field or by using substrates. Recent observations showed that the strength of the Rashba SOC can be enhanced up to $100 \mathrm{meV}$ from 
Gold $(\mathrm{Au})$ intercalation at the graphene-Ni interface 28. A Rashba splitting about $225 \mathrm{meV}$ in epitaxial graphene layers grown on the surface of $\mathrm{Ni}[29$ and a giant Rashba SOC $(\sim 600 \mathrm{meV})$ from $\mathrm{Pb}$ intercalation at the grapheneIr surface 30 are noted in experiments. In view of the above discussion, GNRs can be used as spin-based devices. Consequently a variety of graphene-based spintronic devices have been proposed 31 39, for example, prediction of spin-valve devices based on graphene nanoribbons exhibit giant magnetoresistance (GMR) 33, spin-valve experiment on GNR 34, study of spin polarization and giant magnetoresistance in GNR 35, experiments of GNR as field-effect transistor [36 and p-n junctions [37] using bottom-up fabrication technique and many more [38, 39]. However, most of the studies have been dedicated to making GNR as information storage or attempted to get larger spin polarization. For a complete realization of spintronic applications, a fine control of the spin polarization is highly desirable.

Further, a few of the studies have been dedicated on the transport properties and band structures of graphene nanoribbon in presence of antidots 40 43, which, in essence, is same as our 'cavity'. These studies were basically searching for possible ways to open up energy gaps at the Dirac points to propose an on-off switching mechanism using graphene nanoribbons. In this work, in addition to the charge transport properties being alluded briefly, our central focus is to deliberate on the spin polarized transport in order to explore spintronic application elaborated in the subsequent discussion.

In this letter, we have proposed a possible way of tuning the spin polarization in ZGNR modulated with a circular shaped cavity in presence of Rashba SOC. We have shown that the spin polarized transmission of a two terminal ZGNR can be tuned by varying the radius of the circular cavity. Recent experimental advancement in making nanoscale devices also indicate that our proposed theoretical model can be achieved experimentally. Michael et al. showed that it is possible to realize nanometer-scale pores in graphene by controlled exposure to the focused electron beam of a transmission electron microscope 44, and most importantly they do not evolve over time. Nanolithographic technique 45, template growth [46] technique can be used to achieve desired shape and size of nanopores with great accuracy.

If graphene is grown on a corrugated target substrate, it acquires the morphology of the substrate, leading to ripples 44. This, in turn, enhances local SO coupling 47. For example a $\sim 10 \mathrm{meV}$ SO splitting has been reported in flat $\mathrm{Au}$ monolayer [48, whereas graphene$\mathrm{Au}$ hybridization enhances the Rashba splitting upto 100 $\mathrm{meV}$ [28]. Moreover, intercalation of a $\mathrm{Pb}$ monolayer between graphene and $\operatorname{Ir}(111)$ results into an almost negligible structural corrugation and reduces the overall SO coupling 49. In this work, all the calculations are carried out by assuming a flat GNR (uncorrugated) and thus such corrugated scenario is not taken under consideration.
We organize our paper as follows. In the following section, we present the proposed model and the theoretical formalism for the total transmission and spin polarized transmission using the Green's function technique. Subsequently, we include an elaborate discussion of the results where we have demonstrated cavity effect in ZGNR on the spin polarization, that is, how the spin polarized transmission behaves with the dimension of the system and with the position of the centre of the cavity. We end with a brief summary of our results.

Model and Theoretical formulation. - We consider a zigzag graphene nanoribbon (ZGNR) inscribed with a circular-shaped cavity structure as shown in Fig. 1(top). The length and the width of the ZGNR are

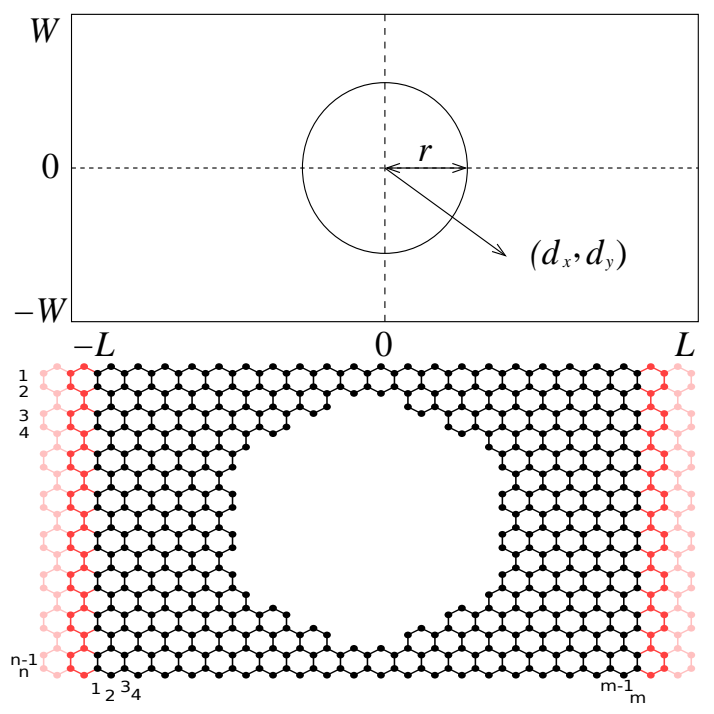

Fig. 1: (Color online). (Top) Schematic diagram for a zigzag graphene nanoribbon with a circular-shaped single cavity. $2 L$ and $2 W$ are the length and the width of the system respectively. The radius of the cavity is denoted by $r$ and the postion coordinate of the centre of the cavity is $\left(d_{x}, d_{y}\right)$. (Bottom) A real setup for calculating the spintronic properties.

$2 L$ and $2 W$ respectively. The radius of the circular-shaped cavity is $r$ and the position of the centre of the cavity is $\left(d_{x}, d_{y}\right)$. We consider a two-terminal setup as shown in Fig. 1 (bottom) in order to calculate the charge and spintronic properties of the ZGNR, where two leads are attached to the central scattering region (ZGNR in this case). The leads are denoted by red colour and are semiinfinite and free from any kind of SO interactions. The Rashba interaction is assumed to be present in the central scattering region only.

The length and the width of the system can be defined in the following way. Since along the length, the system has a zigzag shape and along the width an armchair, we call the system as mZ-nA as shown in Fig. 1(bottom). The system dimension can also be expressed in $\mathrm{nm}$ with the help of the following relations [50], $L_{x}=\frac{\sqrt{3}}{2}(m-1) a, \quad L_{y}=$ $\left(\frac{3}{2} n-1\right) a$, where $a=0.142 \mathrm{~nm}$. Though the nm unit is 
useful to measure sample dimensions experimentally, the $\mathrm{mZ}-\mathrm{nA}$ notation is also helpful in some cases to visualize the system. Thus, throughout this work, we shall use nm unit as well as the mZ-nA notation. However, the radius of the cavity, $r$, is measured in $\mathrm{nm}$.

The tight-binding (TB) Hamiltonian modelled on a ZGNR in presence of Rashba SO interaction can be written as 24,25],

$$
H=-t \sum_{\langle i j\rangle} c_{i}^{\dagger} c_{j}+i \alpha \sum_{\langle i j\rangle} c_{i}^{\dagger}\left(\vec{\sigma} \times \hat{\mathbf{d}}_{i j}\right)_{z} c_{j}
$$

where $c_{i}^{\dagger}=\left(\begin{array}{cc}c_{i \uparrow}^{\dagger} & c_{i \downarrow}^{\dagger}\end{array}\right) \cdot c_{i \sigma}^{\dagger}(\sigma=\uparrow, \downarrow)$ is the creation operator of an electron at site $i$ with spin $\sigma$. The first term is the nearest-neighbor hopping term, with a hopping strength $t=2.7 \mathrm{eV}$. The second term is the nearestneighbor Rashba term which explicitly violates $z \rightarrow-z$ symmetry. $\vec{\sigma}$ denotes the Pauli spin matrices and $\alpha$ is the strength of the Rashba SO interaction. $\hat{\mathbf{d}}_{i j}$ is the unit vector that connects the nearest-neighbor sites $i$ and $j$.

The total transmission coefficient $T$ can be calculated via 51]53,

$$
T=\operatorname{Tr}\left[\Gamma_{L} \mathcal{G}_{R} \Gamma_{R} \mathcal{G}_{A}\right]
$$

$\mathcal{G}_{R(A)}$ is the retarded (advance) Green's function. $\Gamma_{L(R)}$ are the coupling matrices representing the coupling between the central region and the left (right) lead.

Finally, we calculate spin polarized transmission coefficient $P_{s}$ from the relation as [54,

$$
P_{s}=\operatorname{Tr}\left[\hat{\sigma}_{s} \Gamma_{L} G_{R} \Gamma_{R} G_{A}\right]
$$

where, $s=x, y, z$ and $\sigma$ denote the Pauli matrices.

We have studied the charge and spin properties of ZGNR modulated with a circular-shaped cavity structure.

Results and Discussions. - We set the hopping term $t=2.7 \mathrm{eV}$. All the energies are measured in units of $t$. For most of our numerical calculations, we have used KWANT [55. In order to visualize the critical roles of RSOI, and, for the sake of comprehensive analysis we have taken the RSOI strength over a wide range varying from a moderate value $(0.05 t \sim 0.135 \mathrm{eV})$ to a very large one $(0.5 t \sim 1.35 \mathrm{eV})$, which is referred to as a giant Rashba SO coupling.

Before going into the results, there is one important point about the spin polarized transmission that should be mentioned. Since the ZGNR has longitudinal mirror symmetry along $x$ and $z$ axes, the $x$ and $z$ components of the spin polarized transmission are zero. Only the $y$ component has a non-zero value (due to the finite width of the ZGNR along $y$ direction) [38, 39. Hence from now on, we shall call the $y$-component of the spin-polarized transmission coefficient as $P$.

In Fig. 2, the total transmission coefficient $T$ is plotted as a function of the Fermi energy $E$ in the absence of the Rashba SO interaction. We set the dimension of the ZGNR as $9.8 \mathrm{~nm}-8.4 \mathrm{~nm}(81 \mathrm{Z}-40 \mathrm{~A})$. The red colour denotes the plot without a cavity, while the black one denotes the one with cavity with radius $r \sim 2.5 \mathrm{~nm}$. When the cavity is absent in the system, $T$ shows usual discrete step-like feature emphasizing the occurrence of quantum transport at discrete energy values. As the cavity is introduced, the transmission loses its step-like feature and also the magnitude of $T$ gets suppressed. Another important observation is that, in the absence of the cavity a $2 e^{2} / h$ plateau is observed around the zero of the Fermi energy. This $2 e^{2} / h$ plateau plays a vital role in determining the presence of edge states [18,56]. However, this $2 e^{2} / h$ con-
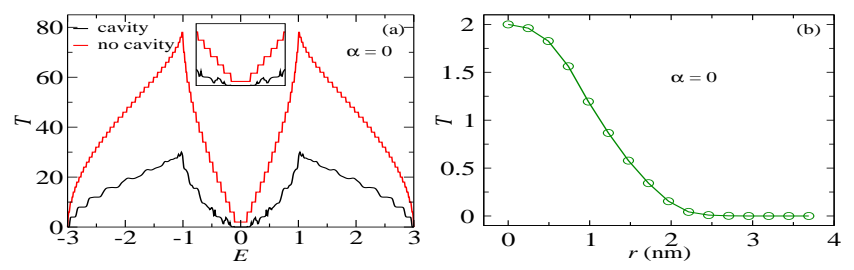

Fig. 2: (Color online). (a) The total transmission coefficient $T$ is plotted as a function of the Fermi energy $E$ in the clean limit. The system dimension is $9.8 \mathrm{~nm}-8.4 \mathrm{~nm}(81 \mathrm{Z}-40 \mathrm{~A})$. The red colour denotes the case without cavity, and, the black one with the cavity where the radius of the cavity is fixed at $r \sim 2.5$ nm. (b) Dependence of $T$ on $r$ setting the Fermi energy $E=0$.

ductance plateau vanishes as we introduce cavity in the system. For better clarity the behaviour of $T$ is plotted, near the zero of the Fermi energy, in the inset in Fig. 2(a). In order to have more information about the vanishing nature of the $2 e^{2} / h$ plateau near the zero of the Fermi energy, we have plotted the total transmission $T$ as a function of the radius of the cavity as shown in Fig. 2 (b). Here, we set the Fermi energy at zero. From the Fig. 2(b), it is observed that $T$ smoothly falls off as we increase the radius of the cavity and vanishes completely after a certain value of $r$. For $r \lesssim 2.5 \mathrm{~nm}$, the system shows metallic behavior, and, for $r \gtrsim 2.5 \mathrm{~nm}$ it acts as an insulator. Thus, a metalto-insulator (MI) transition across the zero Fermi energy can be achieved in a ZGNR using a cavity inscribed. Most importantly, this feature is observed in the absence of any kind of SO interaction. Basically, as we increase the radius of the cavity, the transmission gap is enlarged due to the finite-size effect and as a result the MI transition occurs in the system. The same findings for larger system dimensions have also been done and we have found that though the behaviour of $T$ is not exactly the same as shown in Fig. 2(b) but in all the cases, beyond a certain value of $r$, the system looses the plateau and becomes an insulator.

Let us now include the effect of the Rashba SO interaction and explore the interplay between RSOI and cavity on the spin polarized transport. The spin polarized transmission behaviour in the absence and presence of cavity are presented in Fig. 3. All the plots for the spin polarized transmission are antisymmetric in nature as a function of the Fermi energy $E$ which is due to the electron-hole symmetry of the system. The radius of the cavity is taken as 

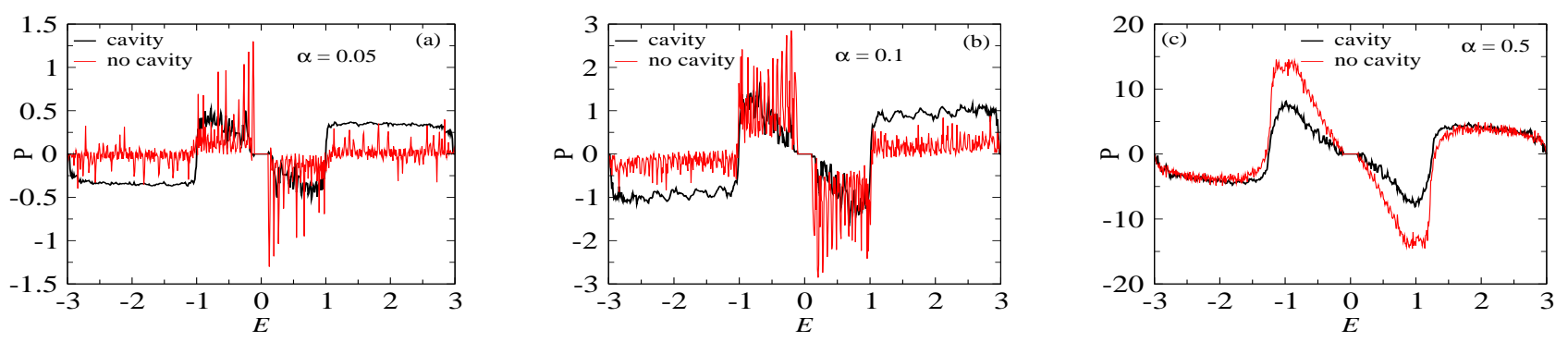

Fig. 3: (Color online). Spin polarized transmission as a function of the Fermi energy $E$ for three different strengths of RSOI $(\alpha)$ considering the system dimension $9.8 \mathrm{~nm}-8.4 \mathrm{~nm}(81 \mathrm{Z}-40 \mathrm{~A})$, where the black and red colored curves correspond to the systems with and without cavity respectively. The radius of the cavity is fixed at $r \sim 2.5 \mathrm{~nm}$.
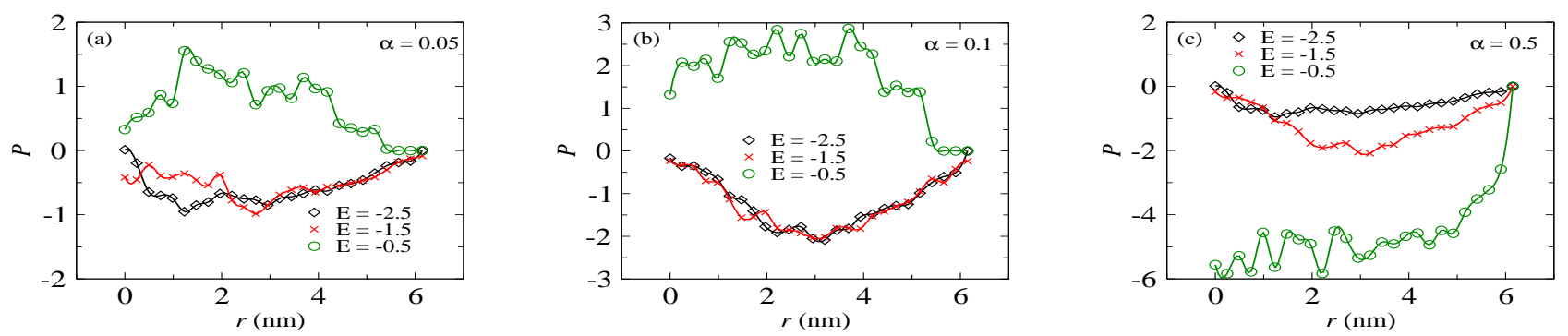

Fig. 4: (Color online). P-r characteristics at three typical energis considering the system size $\sim 15 \mathrm{~nm}-12.6 \mathrm{~nm}(121 \mathrm{Z}-60 \mathrm{~A})$.

$r \sim 2.5 \mathrm{~nm}$ and the system size is kept fixed at $9.8 \mathrm{~nm}-$ $8.4 \mathrm{~nm}(81 \mathrm{Z}-40 \mathrm{~A})$. For smaller values of RSOI, namely, $\alpha=0.05$ and 0.1 , the spin polarized transmission shows reasonably large fluctuations for the cavity-free system as a function of $E$, especially within the range $-1 \leq E \leq 1$ (red lines of Figs. 3(a) and (b)). Whereas the fluctuations get suppressed in the presence of the cavity (black lines of Figs. 3(a) and (b)). Interestingly we see that for these smaller values of RSOI, much larger spin polarization is achieved in presence of cavity beyond the range $E \geq 1$ and $E \leq 1$, which may give an important hint of achieving a high degree of cavity-induced spin polarization, and, to the best of our knowledge, this phenomenon has not been discussed so far in literature. Along with the cavity, the fluctuations get also suppressed with increasing the strength of RSOI, as clearly seen from Fig. 3)(c). Though a smooth curve is obtained, which is always favorable in the context of a physical measurement, but the strength of RSOI in this case (Fig. 3. (c)) is too large and has not been achieved experimentally so far. For our theoretical study, we choose one such large value of $\alpha$ along with other smaller ones, for the sake of a complete analysis. Comparing the spectra given in Fig. 3. one can see that the overall magnitude of $P$ enhances with the rise of $\alpha$.

The above analysis raises an obvious question whether one can tune spin polarized transmission by varying the radius of the cavity. To explore it, let us look into the spectra given in Fig. 4 where $P-r$ characteristics are shown at three typical energies $(E=-2.5,-1.5$ and -0.5 (in units of $t$ ) considering a reasonably large system, compared to the previous one, having system size $15 \mathrm{~nm}-12.6 \mathrm{~nm}$ (121Z-
60A). We choose arbitrarily these typical energies where transmission probability $T$ is sufficiently high $(E=0$ is not be a good choice, as in this case $T$ goes to zero beyond a critical value of $r$, as clearly seen from Fig. 2). Several interesting features are noticed. For different values of $E$, the signs of $P$ are different depending on the dominating spin band (up or down). The most notable aspect is that, as we increase $r$, up to $r \sim 3 \mathrm{~nm}$, the degree of polarization increases, beyond which it starts to decrease. For larger values of $r$ i.e., when $r \approx 6 \mathrm{~nm}, P$ almost drops to zero. This vanishing nature of $P$ at large $r$ is associated with the fact that the number of conducting channels gradually decreases with removing the lattice sites (that is, increasing cavity size), and beyond a critical radius, almost no paths are available for electronic conduction which yields vanishingly small polarization. But below this critical radius that is, where $P$ is finite one may always expect reduced spin polarization with increasing $r$ as spin dependent scattering mechanism, associated with Rashba term, gets weaker with the removal of lattice sites. But the results of Fig. 4 show a non-monotonic nature where $P$ initially increases for smaller $r$, and after reaching to a maximum, it $(P)$ decreases for large values of $r$. There are several possible reasons for that as the polarization effectively depends on which spin channel (up or down) is dominating. One possible reason is the existence of large fluctuations at low cavity sizes, where for a particular energy $E, P$ differs by reasonably large values for two distinct cavity sizes. This difference gradually decreases with large values of $r$, consistent with the suppression of fluctuations, and in that case, reduced values 
of $P$ is observed with $r$, as expected.

From the spectra given in Figs. 3 and 4 we get a clear hint that one can tune $P$ by regulating the cavity size, which is our primary goal of the present analysis. Now, as the value of $P$ depends on the specific choice of the energy $E$, we need to scrutinize further by considering wider range of energy in order to understand the precise role of cavity on spin polarization. In order to do that, in Fig. 5 we show the variation of $P_{\max }$ (maximum value of $P$ ) with $r$ for two different strengths of RSOI (namely $\alpha=0.05$ and 0.1 ) considering the same system size as taken in Fig. 4. $P_{\max }$ is computed by taking the maximum value of $P$ over the complete energy window $[-3: 3]$ (in units of $t$ ). It is seen that $P_{\max }$ decreases with $r$ providing minor fluctuations, and eventually reduces nearly to zero. The decreasing nature can easily be understood, following the results of Figs. 3 and 4, as the scanning is done over the full energy window. The reduction of $P_{\max }$ with $r$ does not actually yield a negative response towards tuning mechanism using cavity, as in the calculation of $P_{\max }$ it takes the maximum value of $P$. While in an experimental situation whenever we talk about possible tuning of $P$ with cavity, we need to fix the Fermi energy at a particular value and there is a high possibility to achieve favorable spin polarization and even much higher polarization than the cavity-free case, as reflected in Figs. 3 and 4 .

For completeness, we have also studied the finite size effects on the behaviour of spin polarized transmission, as shown in Fig. 6] and Fig. 7 respectively. In Fig. 6(a), we

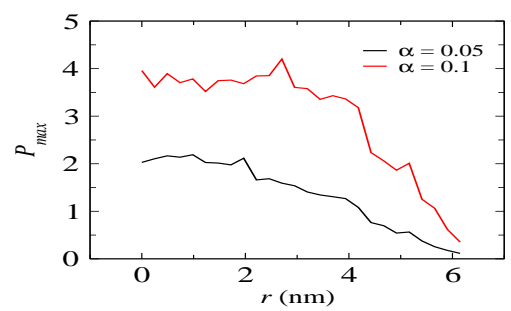

Fig. 5: (Color online). $P_{m a x}-r$ characteristics for two different strengths of RSOI considering the same system size as taken in Fig. 4 .

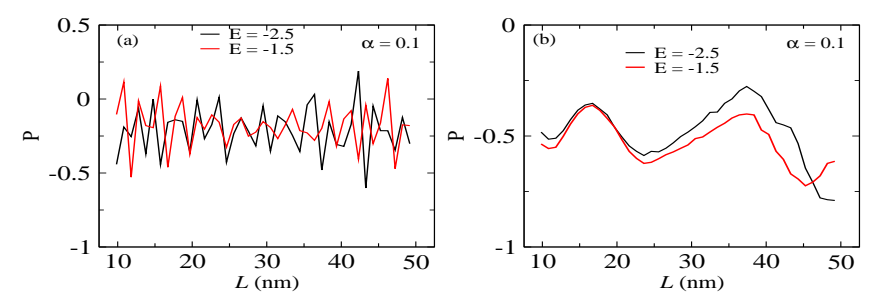

Fig. 6: (Color online). $P$ as a function of the length of the system $L$, considering width of the system as $\sim 16.9 \mathrm{~nm}(80 A)$ and the RSOI strength $\alpha=0.1$. (a) Without cavity, and (b) with cavity having $r \sim 3.7 \mathrm{~nm}$.

have shown the effect of the length (in units $\mathrm{nm}$ ) of the system on $P$ without cavity and in Fig. 6(b) with cavity
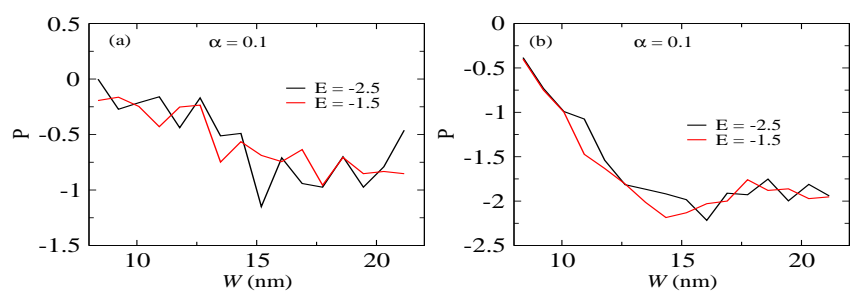

Fig. 7: (Color online). $P$ as a function of the width of the system $W$, taking the length of the system as $\sim 14.8 \mathrm{~nm}(121 \mathrm{Z})$ and the RSOI strength $\alpha=0.1$. (a) Without cavity, and (b) with cavity having $r \sim 3.7 \mathrm{~nm}$.

of radius $r \sim 3.7 \mathrm{~nm}$. The width of the system is taken $\sim 16.9 \mathrm{~nm}(W=80 A)$. We set $\alpha=0.1$. The spin polarized transmission shows large fluctuations in Fig. 6(a). However, in presence of the cavity, the behaviour of $P$ is smooth and oscillating in nature as a function of the length of the system. Further, the effect of the width $W$ (in units of $\mathrm{nm}$ ) of the system on the spin polarized transmission is shown is Fig. 7 Here we have taken the system length as $\sim 14.8 \mathrm{~nm}(121 \mathrm{Z})$ and fixed the Rashba SO interaction strength at $\alpha=0.1$. In the absence of cavity, $P$ shows more fluctuations (Fig. 7(a)) than that with the cavity case (Fig. 7(b). In both the cases, $P$ initially increases with $W$ up to $W \sim 15-16 \mathrm{~nm}$, then it tends to saturate. However, the rate of change of $P$ is more in presence of cavity compared to the cavity free case. Now, as we increase the width of the system the number of modes available for transport increases. As a result, initially $P$ increases with $W$. However, when $W$ is large enough compared to the radius of the cavity, which is the case for $r \sim 3.7 \mathrm{~nm}$, the system tends to behave as that of without cavity.

In all the results discussed so far the centre of the cavity was fixed at the origin or in other words, the position of the centre of the cavity was fixed at $\left(d_{x}, d_{y}=0,0\right)$ (see Fig. 1(top)). It is also important to see how the position of the centre of the cavity affects the spin polarized transport. In other words, in order to answer a rather important question whether there is anything like a most suitable position for the cavity to exist in the system that would yield the maximum spin polarized transmission. To do so, we have considered two different scenario. First, we have varied the $x$-coordinate $\left(d_{x}\right)$ keeping the $y$-coordinate fixed at $y=0\left(d_{y}\right)$. In the second case, we have varied $d_{y}$ along the $x=0$ line. Thus the translation of the cavity in both $x$ and $y$ directions were considered.

The behaviour of the spin polarized transmission as a function of the $x$-coordinate of the centre of the cavity $d_{x}$ (in units of $\mathrm{nm}$ ) and symmetrically with respect to the origin is shown in Fig. 8 for two different values of the Fermi energy, namely $E=-2.5$ and -1.5 in presence of Rashba SO interaction. We have taken three different values of $\alpha$, which are $\alpha=0.05$ (Fig. 8(a)), $\alpha=0.1$ (Fig. 8(b)) and $\alpha=0.5$ (Fig. 8(c)). Here, the centre of the cavity is moving along $y=0$ line and the radius of the cavity is taken as $r=15$. The system dimension is $25 \mathrm{~nm}-8.4 \mathrm{~nm}$ (121Z- 

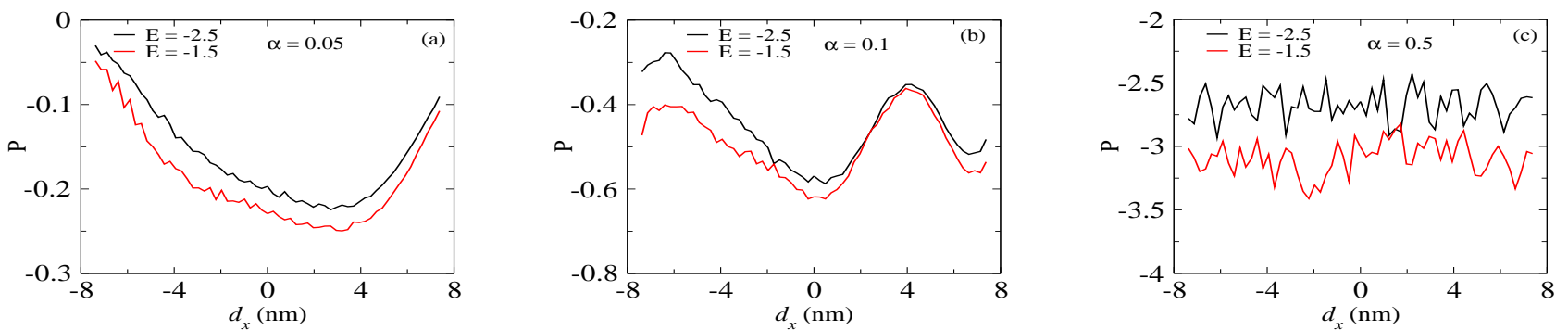

Fig. 8: (Color online). $P$ as a function of position of the centre of the cavity $d_{x}$, considering the dimension of the system as $\sim$ $25 \mathrm{~nm}-8.4 \mathrm{~nm}(201 \mathrm{Z}-40 \mathrm{~A})$. The centre of the cavity is moving along $y=0$ line, and here we choose $r \sim 3.7 \mathrm{~nm}$.
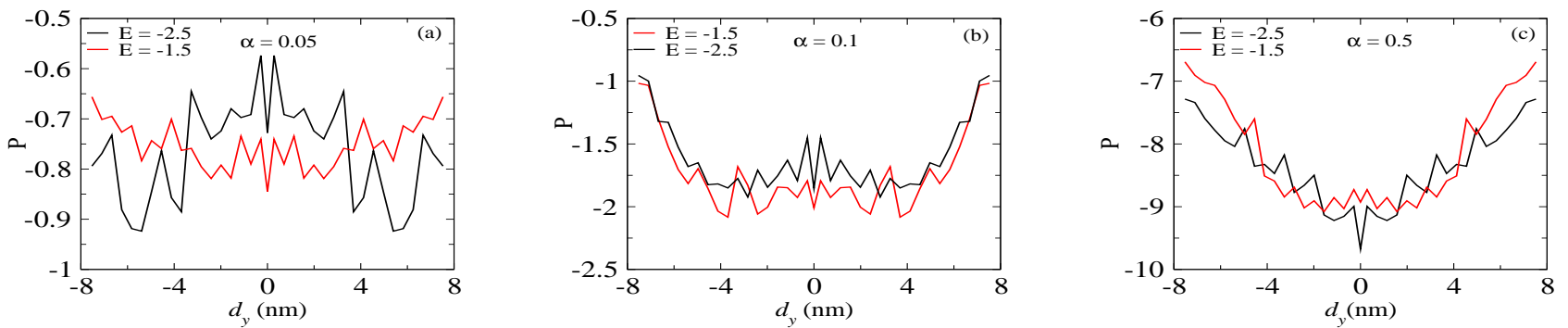

Fig. 9: (Color online). $P$ as a function of position of the centre of the cavity $d_{y}$, taking the system size $14.8 \mathrm{~nm}-21.2 \mathrm{~nm}$ (121Z-100A). The centre of the cavity is moving along $x=0$ line, and we set $r \sim 2.5 \mathrm{~nm}$.

40A). As shown in Fig. 8(a), for $\alpha=0.05, P$ increases as the cavity moves from the left side of the system to the right and attains a maximum value around $d_{x} \approx 4 \mathrm{~nm}$. Subsequently $P$ starts to decrease as the cavity moves towards the right end of the system. For $\alpha=0.1$ (Fig. 8(b)), $P$ shows little oscillatory behavior as a function of $d_{x}$ and the maximum value of $P$ is observed around $d_{x}=0$. For higher values of $\alpha$, that is, $\alpha=0.5$ (Fig. $8(\mathrm{c})$ ), $P$ does not have any regular behaviour as a function of $d_{x}$. This points towards possibilities of significant interplay between the position of the cavity (in addition to the radius as discussed above) and the strength of the Rashba interaction with regard to its spin polarized transport properties.

The behavior of the spin polarized transmission as a function of the $y$-coordinate of the centre of the cavity $d_{y}$ (in units of $\mathrm{nm}$ ) is shown in Fig. 9. Here, the cavity is translated along the $x=0$ line. The radius of the cavity is taken as $r \sim 2.5 \mathrm{~nm}$ and the dimension of the system is $14.8 \mathrm{~nm}-21.2 \mathrm{~nm}(121 \mathrm{Z}-100 \mathrm{~A})$. For the three different values of $\alpha$, viz, $\alpha=0.05$ (Fig. 9(a)), $\alpha=0.1$ (Fig. 9(b)) and $\alpha=0.5$ (Fig. 9(c)), $P$ shows symmetric behaviour as a function of $d_{y}$ about $d_{y}=0$ due to the symmetry of the position of the cavity about the $x=0$ line. The magnitude of $P$ is higher for higher values of $\alpha$, which is obvious.

From the study of the behavior of spin polarized transmission as a function of the positions of the centre of the cavity, it is difficult to comment on the optimized position of the cavity, so that one can get large spin polarized transmission. This is apparent from the fact that there is no regular feature of $P$ as seen from Fig. 8 and Fig. 9 , However, from Fig. 9, we can at least recommend that, it is prudent not to put the cavity at far left or far right edge of the system, since at these positions, $P$ has lower values compared to other intermediate locations.

Conclusion. - In summary, we have studied briefly the charge transmission and more elaborately spin polarized transport properties in a two-terminal ZGNR modulated with a circular-shaped cavity in presence of RSOI. The charge transmission shows interesting feature in presence of the cavity. In particular, we notice a cavityinduced MI transition in the interacting free system. From the detailed analysis of spin polarized transport we have shown that by varying the radius of the cavity, one can tune the spin polarized transmission efficiently in a twoterminal ZGNR. All the results are obtained for fairly large dimensions of the system. Moreover, we have also studied the effect of the system dimension, such as the length and width of the system on the spin polarized transmission. The spin polarized transmission oscillates with the length of the system and for larger values of the width it tends to saturate. However, the main focus of this work, that is the tuning of the spin polarized transmission, shows qualitatively similar behaviour for larger dimensions of the system. The position of the centre of the cavity is also an important parameter for tuning the spin polarized transmission. In order to achieve higher spin polarized transmission, we issue a word of caution that one should not put the cavity at the extreme left or at the extreme right edge of the system.

Considering the success of fabricating 'holey Graphene' 57] seamlessly, our work may render a new direction to engineer tunable spin polarized trans- 
port properties of GNRs via cavities of different shapes and sizes.

SB thanks SERB, India for financial support under the grant F. No: EMR/2015/001039.

\section{REFERENCES}

[1] S. A. Wolf et al., Science 294, 5546 (2001).

[2] I. Žutić, J. Fabian, and S. Das Sarma, Rev. Mod. Phys. 76, 2 (2004)

[3] K. S. Novoselov et al., Science 306, 666 (2004).

[4] Luis E. Hueso et al., Nature 445, 410 (2007).

[5] N. Tombros, C. Jozsa, M. Popinciuc, H. T. Jonkman and B. J. van Wees, Nature 448, 571 (2007).

[6] P. J. Zomer, M. H. D. Guimarães, N. Tombros, and B. J. van Wees, Phys. Rev. B 86, 161416(R) (2012).

[7] T.- Y. Yang et al., Phys. Rev. Lett. 107, 047206 (2011).

[8] Wei Han and R. K. Kawakami, Phys. Rev. Lett. 107, 047207 (2011).

[9] A. H. C. Neto, F. Guinea, N. M. R. Peres, K. S. Novoselov, and A. K. Geim, Rev. Mod. Phys. 81, 109 (2009).

[10] Y. Zhang, Y.-W. Tan, H. L. Stormer, and P. Kim, Nature 438, 201 (2005).

[11] V. P. Gusynin and S. G. Sharapov, Phys. Rev. Lett. 95 , 146801 (2005).

[12] E-J. Kan, Z. Li, J. Yang, and J. G. Hou, Appl. Phys. Lett. 91, 243116 (2007).

[13] X. Lin and J. Ni, Phys. Rev. B 84, 075461 (2011).

[14] X. Du, I. Skachko, A. Barker, and E. Y. Andrei, Nat. Nanotech. 3491 (2008).

[15] K. I. Bolotin , K. J. Sikes , J. Hone, H. L. Stormer, and P. Kim, Phys. Rev. Lett. 101096802 (2008).

[16] J. C. Meyer, A. K. Geim, K. S. Novoselov, T. J. Booth, and S. Roth, Nature London 446,60 (2007).

[17] S. V. Morozov et al., Phys. Rev. Lett. 97, 016801 (2006).

[18] K. Wakabayashi, M. Fujita, H. Ajiki and M. Sigrist, Phys. Rev. B 59, 8271 (1999).

[19] R. Saito, M. Fujita, G. Dresselhaus, and M. S. Dresselhaus, Appl. Phys. Lett. 60 , 2204 (1992)

[20] K. Nakada, M. Fujita, G. Dresselhaus, and M. S. Dresselhaus, Phys. Rev. B 54, 17954 (1996).

[21] O. V. Yazyev and M. I. Katsnelson, Phys. Rev. Lett. 100, 047209 (2008).

[22] O. V. Yazyev, Nano Lett. 8, 1011 (2008).

[23] G. Cantele, Y.-S. Lee, D. Ninno, and N. Marzari, Nano Lett. 9, 3425 (2009).

[24] C. L. Kane and E. J. Mele, Phys. Rev. Lett. 95, 226801 (2005).

[25] C. L. Kane and E. J. Mele, Phys. Rev. Lett. 95, 146802 (2005).

[26] Y. Yao, F. Ye, X.L. Qi, S.C. Zhang, and Z. Fang, Phys. Rev. B 75, 041401 (2007).

[27] J. C. Boettger and S. B. Trickey, Phys. Rev. B 75, 121402 (2007).

[28] D. Marchenko et al., Nat. Commun. 3, 1232 (2012).

[29] Y. S. Dedkov, M. Fonin, U. Rüdiger, and C. Laubschat, Phys. Rev. Lett. 100, 107602 (2008).

[30] F. Calleja et al., Nat. Phys. 11, 43 (2015).
[31] F. Schwierz, Nat. Nanotech. 5, 487 (2010).

[32] M. Zeng, L. Shen, M. Zhou, C. Zhang, and Y. Feng, Phys. Rev. B 83, 115427 (2011).

[33] W. Y. Kim and K. S. Kim, Nat. Nanotech. 3, 408 (2008).

[34] C. Józsa, M. Popinciuc, N. Tombros, H. T. Jonkman, and B. J. van Wees, Phys. Rev. Lett. 100, 236603 (2008).

[35] Y. -T. Zhang, H. Jiang, Q.-f. Sun, and X. C. Xie, Phys. Rev. B 81, 165404 (2010).

[36] P. B. Bennett et al., Appl. Phys. Lett. 103, 253114 (2013).

[37] J. Cai et al., Nat. Nanotech. 9, 896 (2014).

[38] L. Chico, A. Latge, and L. Brey, Phys. Chem. Chem. Phys. 17, 16469 (2015).

[39] Q. Zhang, K. S. Chan, and J. Li, Phys. Chem. Chem. Phys. 19, 6871 (2017)

[40] Y. Lv, S. Chang, H. Wang, J. He, and Q. Huang, Carbon 101, 143 (2016).

[41] A. J. M. Giesbers, E. C. Peters, M. Burghard, and K. Kern, Phys. Rev. B 86, 045445 (2012).

[42] L. Rosales, M. Pacheco, Z. Barticevic, A. León, A. Latgé, and P. A. Orellana, Phys. Rev. B 80, 073402 (2009).

[43] Mads L. Trolle, Ulrik S. Møller, and Thomas G. Pedersen, Phys. Rev. B 88, 195418 (2013).

[44] M. D. Fischbein and M. Drndić, Appl. Phys. Lett. 93, 11 (2008).

[45] Q. Zhang et al., Nano Res. 6, 478 (2013).

[46] N. S. Safron, M. Kim, P. Gopalan, and M. S. Arnold, Advanced Materials 24, 8 (2012).

[47] A. H. Castro Neto and F. Guinea, Phys. Rev. Lett. 103, 026804 (2009).

[48] A, Varykhalov, A. et al., Phys. Rev. Lett. 101, 157601 (2008).

[49] M. M. Otrokov et al., 2D Mater. 5035029 (2018).

[50] S. Ganguly, S. Basu, and S. K. Maiti, Superlattices and Microstructures 120, 650 (2018).

[51] C. Caroli, R. Combescot, P. Nozieres, and D. Saint-James, J. Phys C: Solid State Phys. 4, 916, (1971).

[52] D. S. Fisher and P. A. Lee, Phys. Rev. B 23, 6851 (1981).

[53] S. Datta, Electronic transport in Mesoscopic systems, University press (Cambridge), (1995).

[54] P.-H. Chang, F. Mahfouzi, N. Nagaosa, and B. K. Nikolic, Phys. Rev. B 89, 195418 (2014).

[55] C. W. Groth, M. Wimmer, A. R. Akhmerov, and X. Waintal, New J. Phys. 16, 063065 (2014).

[56] S. Ganguly and S. Basu, Mater. Res. Express 4, 11 (2017).

[57] Y. Lin and Y. Liao, Z. Chen, and J. W. Connell, Mater. Res. Lett. 5, 4 (2017). 